\title{
Maternal Near Miss Review: A Way Forward
}

\section{Purandare C. $\mathbf{N}$.}

Published online: 13 August 2013

(C) Federation of Obstetric \& Gynecological Societies of India 2013

\section{Introduction}

India has made significant progress in reducing its maternal mortality rate (MMR) from 254 (SRS 2004-06) to 212 (SRS 2007-09) [1] per hundred thousand live births (LB); however, there is a long way to go on this journey to meet the millennium development goals. Maternal mortality (MM) is frequently described as "Just The Tip of The Iceberg" alluding that there is a vast base to the iceberg in the form of maternal near miss (MNM) i.e. maternal morbidity which has remained largely undescribed. In 2009, Roost et al. [2] reported MMR as 187/100,000 LB and MNM as 50/1,000 LB, with a relatively low mortality index (MI) of $3.6 \%$.

Large differences were found among countries on the incidence of MNM because of the different settings and variation in the criteria used to define the MNM. In 2009, World Health Organization (WHO) defined a MNM case as "a woman who nearly died but survived a complication that occurred during pregnancy, childbirth or within 42 days of termination of pregnancy" [3].

About two decades ago, in low MM settings, MNM was found to be a more useful indicator of obstetric care than MM alone. In recent years, evidence from developing

Purandare C. N. ( $₫)$, Ex-Professor

Department of Obstetrics \& Gynaecology, Grant Medical

College, Purandare Griha 31/C, Dr. N.A. Purandare Marg,

Mumbai 400 007, India

e-mail: dr.c.n.purandare@gmail.com country settings, analyzing these near-miss cases, helps in understanding health system failures in relation to obstetric care and addressing them. Reviews of MNM cases are likely to yield useful information as MNM cases are not as rare as maternal deaths, they have the same pathways which lead to deaths, and provide information regarding care received and possible means of prevention, particularly in facilities where deaths are uncommon and where events associated with deaths may not be typical. As the woman survives, near-miss reviews may be seen as less threatening than death reviews for the teams who report them.

Filippi et al. [4] classified disease-specific criteria for identifying MNM cases, and they are based on five main categories of complications at term. They are hemorrhage resulting into shock, emergency hysterectomy and blood transfusion, pregnancy-induced hypertensive disorders includes eclampsia and severe pre-eclampsia, dystocia i.e. uterine rupture and impending rupture, infections causing hyper/hypothermia and/or clear source of infection with clinical signs of shock, and anemia hemoglobin levels below or equal to $50 \mathrm{~g} / \mathrm{l}$ or clinical signs of severe anemia.

WHO recommended three different approaches of selection criteria for identification of MNM cases [5]. They are disease specific, management based and organ dysfunction. Disease-specific morbidities are severe preeclampsia, eclampsia, severe hemorrhage, severe sepsis and uterine rupture, however have too low threshold of morbidity to be called near misses. Management based criteria are according to specific intervention made e.g. 
admission to ICU, procedure such as obstetric hysterectomy, massive blood transfusion, intubation and ventilation. In 2004, Anwari et al. [6] reported that 99 obstetric admissions to ICU were represented by $0.2 \%$ of all deliveries and $1.6 \%$ of all ICU admissions. Most of the patients were young. The two most common indications for admitting obstetric patients to ICU were hemorrhage and hypertension. They concluded that invasive hemodynamic monitoring and ventilatory support were the two main interventions and improving quality of care before and after admission to ICU may reduce maternal morbidity. In $\mathrm{UK}$, at tertiary care centre, there were 15 cases of emergency peripartum hysterectomy in 31,079 deliveries, giving the incidence of 0.48 per 1,000 . Haemorrhage due to placenta praevia especially in repeat $\mathrm{C}$ section was the main indication for emergency peripartum hysterectomy (47\%) [7] while in Turkey at tertiary care centre, the incidence of emergency peripartum hysterectomy was 0.67 in 1,000 deliveries and hemorrhage due to uterine atony was the main indication for emergency hysterectomy (57.1\%).[8]. Organ system dysfunction is based on apparent clinical diseases, clinical markers and management needed. Aim is toward correction of that organ dysfunction to arrest near-miss progression to maternal death e.g. cardiovascular dysfunction, respiratory dysfunction, renal dysfunction, coagulation dysfunction, hepatic dysfunction, neurological dysfunction, uterine dysfunction. The organ system dysfunction based approach is considered as "the most promising frame" for establishing a standard set of criteria as it provides more reliable identification of real near-miss cases however, it needs a minimum level of care and basic critical care monitoring facilities hence, cannot be used in low resource settings. In 2011, Cecatti et al. [9] studied 673 women admitted in ICU with severe maternal morbidity and could identify 194 cases of MNM and the 18 maternal deaths by WHO criteria. They concluded that with the WHO criteria for MNM they were able to identify almost all cases of death and organ failure.

Taking into consideration all the literature data disorders, symptoms, signs, laboratory investigations, and interventions, new comprehensive criteria were developed for use in the pilot. The criteria were divided into four groups: 1 pregnancy-specific disorder, 2 preexisting disorder aggravated during pregnancy, 3 pregnancy-specific medical disorder, and 4 incidental and accidental causes in pregnancy.

Important MNM indicators as defined by WHO are severe maternal outcome ratio (SMOR) and MI. SMOR refers to the number of women with life-threatening conditions (MNM + MD) per 1,000 LB. This indicator gives an estimate of the amount of care and resources that would be needed in an area $[\mathrm{SMOR}=(\mathrm{MNM}+\mathrm{MD}) / 1,000 \mathrm{LB}]$.
MI refers to the number of maternal deaths divided by the number of women with life-threatening conditions expressed as a percentage $[\mathrm{MI}=\mathrm{MD} /(\mathrm{MNM}+\mathrm{MD})]$. The higher the index the more the women with lifethreatening conditions die indicating low quality of care, whereas the lower the index the fewer women with lifethreatening conditions die indicating better quality of care.

In India, MNM Review Pilot Project was implemented under the guidance of the Maternal Health division of Government of India, Ministry of Health and Family Welfare, Federation of Obstetric and Gynaecological Societies of India (FOGSI), Mahatma Gandhi Institute of Medical Sciences (MGIMS), Systems and Program Management, 06 Medical colleges through support and funding provided by LOGIC program of FIGO [ LOGIC: Leadership in Obstetrics and Gynecology for Impact and Change; FIGO: International Federation of Gynecology and Obstetrics].

The pilot project comprised twelve phases and the work is in progress. In the Indian context, the following definition of MNM was used for the pilot.

A woman who survives severe life threatening conditions, either after receiving emergency medical/ surgical interventions or otherwise; during pregnancy, abortion, childbirth or within 42 days of pregnancy termination.

Preliminary analysis of data showed that a total of 160 MNM cases were reported during the period JulyNovember 2012. As reporting of MNM case is less threatening, more number of cases were reported and information in the tools was diligently filled up by the teams. MNM cases of $62.5 \%$ occurred in antenatal phase, $23.8 \%$ in postnatal phase, $3.8 \%$ in intranatal phase, $8.1 \%$ abortion phase, and $1.9 \%$ in post-abortion phase. Hemorrhage was the major complication contributing to $46.9 \%$ of all the cases with intrapartum bleeding and atonic postpartum hemorrhage as 1.9 and $23.7 \%$, respectively. Hypertensive disorders lead to $23.1 \%$ MNM cases. Eighteen cases were reported in each group of severe preeclampsia and eclampsia. Sepsis was observed in $4.4 \%$ cases. Medical disorders also contributed to major causes of MNM with anemia in $8.75 \%$, hepatitis in $6.25 \%$.

Antenatal care (ANC) continues to be an issue. $61.2 \%$ MNM cases did not receive proper ANC. Proper referral was a challenge as in $66.9 \%$ cases, referral was delayed or was not done properly. In $77.7 \%$ cases, partograph was not used. In $28.8 \%$ MNM cases, a single system, and in $61.9 \%$ MNM cases, multiple systems were involved. Duration of illness to admission with mean time of $48.41 \mathrm{~h}$, duration of onset of near-miss morbidity in admitted patient of $12.65 \mathrm{~h}$ contributed to first and second delays. 
Lack of education $(57.5 \%)$, poor understanding of complications and risk factors in pregnancy and required medical interventions $(19.4 \%)$, previous poor experience of health care $(38.8 \%)$, financial implications $(70.1 \%)$, and inadequate referral systems $(33.1 \%)$ were contributory factors for MNM cases.

In summary, any pregnant woman can develop lifethreatening complications with little or no advance warning. All women need access to quality maternal health services that can diagnose and manage life-threatening complications. In developing countries, women's low economic status, poor obstetric services, and lack of emergency transfers during labor contribute significantly to MM and MNM cases; therefore, emphasis on implementation of Emergency Obstetric Care, Janani Surakhsha Yojana, referral transport like services are essential. Maternal death and MNM cases are the indicators of the quality of health care provided. Obstetric ICU set up with a team approach consisting of treatment by obstetricians, intensive care specialists, and anesthesiologists are essential to save a maternal life. The most vital purpose of the near-miss approach is to improve clinical practice and reduce preventable morbidity and mortality through the use of best evidence-based practices.

\section{References}

1. Special Bulletin on Maternal Mortality in India 2007-09 Sample Registration System (SRS) office of Registrar General, India, June 2011.

2. Rööst M, Altamirano VC, Liljestrand J, et al. Priorities in emergency obstetric care in Bolivia-maternal mortality and near-miss morbidity in metropolitan La Paz. BJOG. 2009;116(9):1210-7.

3. Report on the World Health Organization Working Group on the Classification of Maternal Deaths and Severe Maternal Morbidities. Geneva: World Health Organization, 2009.

4. Filippi V, Ronsmans C, Gohou V, et al. Maternity wards or emergency obstetric rooms? Incidence of near-miss events in African hospitals. Acta Obstet Gynecol Scand. 2005;84(1):11-6.

5. Say L, Pattinson RC, Gulmezoglu AM. WHO systematic review of maternal morbidity and mortality: the prevalence of severe acute maternal morbidity (near miss). Reprod Health. 2004;1:3.

6. Anwari JS, Butt AA. Al-Dar MA Saudi Obstetric admissions to the intensive care unit. Med J. 2004;25(10):1394-9.

7. Selo-Ojeme DO, Bhattacharjee P, Izuwa-Njoku NF, et al. Emergency peripartum hysterectomy in a tertiary London hospital. Arch Gynecol Obstet. 2005;271(2):154-9.

8. Güngördük K, Yildirim G, Dugan N, et al. Peripartum hysterectomy in Turkey: a case-control study. J Obstet Gynecol. 2009;29(8): $722-8$.

9. Cecatti JG, Souza JP, Oliveira Neto AF, et al. Pre-validation of the WHO organ dysfunction based criteria for identification of maternal near miss. Reprod Health. 2011;2(8):22. 